\title{
Formation flying control for satellites: anti-windup based approach
}

\author{
J. Boada, C. Prieur, S. Tarbouriech, C. Pittet, and C. Charbonnel
}

\begin{abstract}
Control theory has significantly evolved in the field of the nonlinear control. However, the methods used in the aerospace industry lie usually on linear techniques applied to linearized models. The increasing requirements in terms of operational reliability and performance ask for the development of new control techniques more complex in order to meet the new demands. Therefore the industry is moving to the modern control theory looking for new nonlinear approaches. In particular, actuators saturation represents a nonlinear phenomenon common in almost all physical applications. This can then lead to performance degradation, limit cycle appearance, non-desired equilibrium conditions and even system instability. The objective of this chapter is to adapt and develop the anti-windup compensator design to the control with high precision for the angular and the linear axes of a satellite. In the aerospace application field, this situation meets with the drag-free or the formation flying missions. These missions use high precision thrusters as actuators whose capacity appears to be critically low. Moreover thrusters have a particular modeling. Allocation functions adapted to the anti-windup design are then explored. In addition considering the current state of the art of the anti-windup design, there is a strong necessity of using symmetrizing techniques for the saturation. The main objective of this work consists in applying the developed tools on an aerospace study case. As an example, a complete methodology is proposed to control a formation flying mission controlling both attitude and relative position.
\end{abstract}

Key words: Saturating thrusters, anti-windup design, control, optimization

J. Boada is with Albatros Aeronautics, Vitoria, Spain, e-mail: josep.boada@albaero.com . C. Prieur is with Gipsa-lab, Department of Automatic Control, Grenoble Campus, 11 rue des Mathématiques, BP 46, 38402 Saint Martin d'Hères Cedex, France, e-mail: christophe.prieur@gipsa-lab.fr·S. Tarbouriech is with CNRS, LAAS, 7 avenue du Colonel Roche, F-31400 Toulouse, France. Univ de Toulouse, LAAS, F-31400 Toulouse, France, e-mail: tarbour@laas.fr . C. Pittet is with Centre National d'Etudes Spatiales (CNES), Toulouse, France, e-mail: christelle.pittet@cnes.fr ·C. Charbonnel is with Thales Alenia Space, Cannes, France, email: catherine.charbonnel@thalesaleniaspace.com 


\section{Introduction}

Formation flying control problem has been an important field of research since the 1990's. Several possible applications in the space exploration domain make this field very interesting $[15,24,1]$. In these kinds of missions one seeks to control the formation with a fine precision in both attitude and relative position. Consequently, the actuator is based on a precise propulsive system. However, these kinds of actuators have a limited capacity which cannot be exceeded.

The control limitation due to the constraints of the actuators' maximum capacity represents a nonlinear phenomenon common in almost all physical applications. Traditionally, a classical solution, at least in industry, consists in imposing important margins in order to prevent the actuators from reaching their maximum capacity, that is, to avoid saturation. In that manner, one tries to ensure the linearity of the system. However this a posteriori validation is insufficient because, non-nominal disturbances, transitions between operational modes, and the presence of system failures can force the actuators to reach their limits. Actuator saturation can then lead to performance degradation, limit cycle appearance, non-desired equilibrium conditions and even system instability [2, 18, 19, 26, 27].

The nonlinear techniques dealing with saturation can be classified in two main research lines. The first one seeks to introduce the nonlinear saturation in the synthesis process of the controller. The second one introduces an extra layer to the existing linear controller, accounting for the nonlinearities. This strategy, also called anti-windup design, allows the designer to keep the existing linear controller (already validated) and only to introduce a compensator which is only active when the nonlinearity arises $[17,31,20,14,29,27,33]$. The design of such a compensator is generally cast in a static optimization problem of the controller parameters. Accompanying the development of semi-definite programming and convex optimization [10], the synthesis problem can be formulated as the optimization of a multi-objective criterium (corresponding to closed-loop stability and performance specifications) subject to matrix inequalities constraints associated to the dynamical system.

The objective of this chapter is to depict how the anti-windup compensator design can be adapted to high precision control of the linear and angular displacements of a satellite. Consequently, in formation flying control problem, the introduction of the anti-windup becomes an interesting technique to ensure the mission requirements and its reliability. Moreover, let us point out that space missions involving thrusters as actuators are modeled in a particular way. The control variables of the physical systems and the action provided by thrusters are not the same. An allocation function is included in the modeling of the actuators to distribute the control among the actuators $[11,22,4]$. In the classical linear approach this function can be omitted, however, when the saturation of the actuators arises, its behavior has to be considered in the control design [4]. Allocation functions adapted to the antiwindup design have to be explored. Moreover the presence of thrusters introduces a peculiar formulation of the saturation function. The saturation presents asymmetric bounds with the minimum equal to zero. Considering the current state of the art of 
the anti-windup design, there is a strong necessity of using symmetrizing techniques for the saturation. The symmetrizing procedure and the allocation function defined are put along with the anti-windup design to be applied in a spacecraft mission configuration.

For anti-windup study purposes, the formation flying problem can be described by a block diagram as presented in Figure 1. If $y_{p}$ appears as attitude and/or relative position, the control loop would be illustrative of a formation flight configuration.

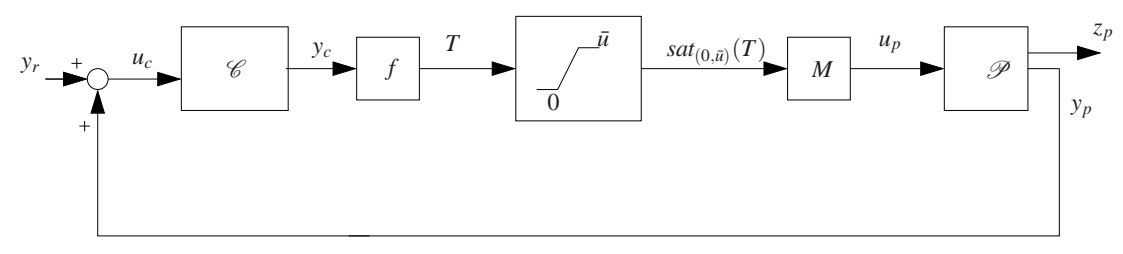

Fig. 1: Control loop block diagram.

Remark 1. $y_{r}=0$ for consistency reasons without loss of generality.

In this chapter a formation flying control problem is modeled and solved. This is the relative position of a two satellites formation. We prefer to focus on this problem to ease the presentation of the anti-windup methods. However this reduction is not a necessary simplification, and a more complete multi-variable system with a coupling of the attitude and the relative position of two satellites could be tackled. For more details on this last case see [6], where the saturation effects are studied and some anti-windup methods are developed. Let us emphasize that the anti-windup design problem considered corresponds to a multiobjective control optimization problem, which consists in minimizing some performance criteria (closed-loop $\mathscr{L}_{2}$-gain, fuel consumption) and maximizing the size (via the choice of optimal criteria) of the state space region of safe operation (guaranteed stability around the origin, attainability of the performance level), in presence of two hard nonlinear phenomena as the presence of both actuator saturation and allocation function. Thus this kind of mathematical programming approach can be viewed as complementary to on-line optimization-based methods, which require in general a high computational effort.

The chapter is organized as follows. First, in Section 2, the model of the relative position between two satellites is presented. Only the control of an axis is considered. Then, in Section 3 the following anti-windup techniques are applied to the relative position control: the Direct Linear Anti-Windup (DLAW), the Model Recovery Anti-windup (MRAW) and the Extended Model Recovery Anti-windup (EMRAW). Simulations illustrate the benefits provided by the anti-windup compensators. Some concluding remarks end the chapter. 


\section{Relative position control}

\subsection{Relative position plant model}

The first relative dynamics to be described is the relative position between two satellites along the z-axis. Let us consider two satellites and two frames fixed to each satellite. $\mathscr{F}_{\text {sat } 1}$ is the first satellite associated frame and $\mathscr{F}_{\text {sat } 2}$ the second satellite associated frame. From the third theorem of the rigid body dynamics, and assuming that the satellite is a point-mass system, the acceleration of a rigid body is proportional to the sum of external forces:

$$
\ddot{z}_{i}=m_{i}^{-1} \sum\left(F_{i}\right)
$$

where $z_{i}$ is the displacement on the $z$-axis of satellite $i$. Hence, $\ddot{z}_{i}$ denotes the acceleration on this axis. $m_{i}$ denotes the mass of satellite $i$ and $\sum\left(F_{i}\right)$ stands for the sum of external forces on satellite $i$.

The control objective is to cancel the lateral position error on the $z$ coordinate between the satellites (see Figure 2). Therefore, the relative dynamics can be described applying (1) to the difference of the $z_{i}$ coordinate with $i=1,2$, that is $\Delta z=z_{1}-z_{2}$. The control objective is then to satisfy $\Delta z=0$. Denoting $\sum\left(F_{i}\right)$ by $F_{i}$ for consistency reasons, it yields $\Delta \ddot{z}=-m_{2}^{-1} F_{2}+m_{1}^{-1} F_{1}$.

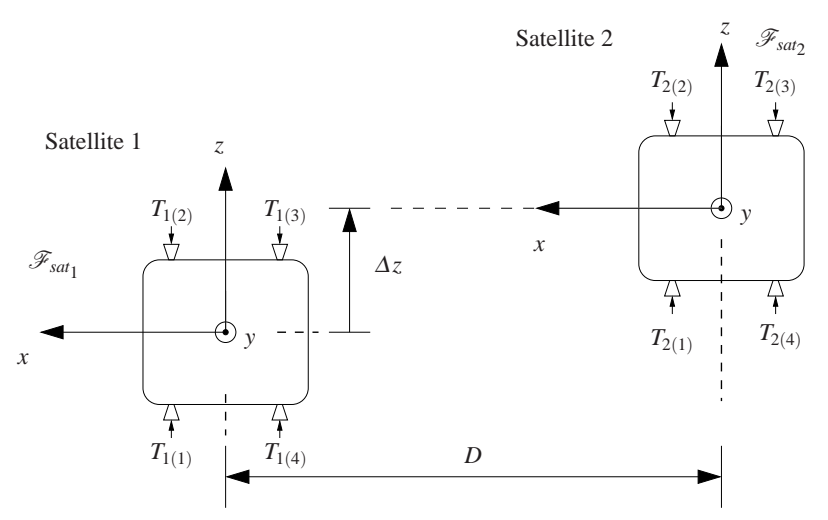

Fig. 2: Relative position control configuration.

The state space representation reads:

$$
\left\{\begin{array}{l}
\dot{x}_{p}=A_{p} x_{p}+B_{p} u_{p}=\left[\begin{array}{ll}
0 & 1 \\
0 & 0
\end{array}\right]\left[\begin{array}{l}
\Delta z \\
\Delta \dot{z}
\end{array}\right]+\left[\begin{array}{cc}
0 & 0 \\
m_{1}^{-1} & -m_{2}^{-1}
\end{array}\right] u_{p} \\
y_{p}=C_{p} x_{p}=\left[\begin{array}{ll}
1 & 0
\end{array}\right] x_{p}=\Delta z \\
z_{p}=C_{z} x_{p}=\left[\begin{array}{ll}
1 & 0
\end{array}\right] x_{p}=\Delta z
\end{array}\right.
$$


where the state variables included in the state vector $x_{p}$ are the relative position $(\Delta z)$ and the relative velocity $(\Delta \dot{z}), u_{p}=\left[\begin{array}{ll}F_{1} & F_{2}\end{array}\right]^{\prime}$ is the control input, $y_{p}=\Delta z$ is the measured output and $z_{p}=\Delta z$ is the regulated output.

\subsection{Relative position controller}

A centralized controller is used. This means that a unique controller takes the measurements from all satellites in the formation and computes a vector $y_{c}$ which contains the control output of each satellite. The controller is described through a state space representation:

$$
\left\{\begin{array}{l}
\dot{x}_{c}=A_{c} x_{c}+B_{c} u_{c} \\
y_{c}=C_{c} x_{c}+D_{c} u_{c}
\end{array}\right.
$$

In the relative position problem, the controller is an 1-input 2-outputs (Single Input Multiple Output or SIMO) linear system with a 5 dimension state vector. The controller input is $u_{c}=y_{p}=\Delta z$ and its outputs are $y_{c}=\left[\begin{array}{ll}y_{c 1} & y_{c 2}\end{array}\right]^{\prime}=\left[\begin{array}{ll}F_{c 1} & F_{c 2}\end{array}\right]^{\prime} . F_{c 1}$ (resp. $F_{c 2}$ ) stands for the controller output for the first (resp. second) satellite.

\subsection{Relative position actuator model}

The satellite formation is actuated by a propulsive system composed of 4 proportional thrusters on each of the two satellites. To apply the required control forces $\left(F_{1}\right.$ and $\left.F_{2}\right)$ using this propulsive system, thruster management functions have to be introduced in the control loop. These functions are composed by an allocation function that transforms the required control efforts $\left(F_{c 1}, F_{c 2}\right)$ into thrusters forces, and an influence matrix that transforms the thruster outputs into forces applied on the system. Moreover, the actual forces delivered by each thruster are saturated. The general expression for the actuator is given as follows:

$$
u_{p}=\operatorname{Msat}_{(0, \bar{u})}\left(f\left(y_{c}\right)\right)
$$

where $M$ is the influence matrix for each satellite, sat is the thruster saturation, and $f$ is the allocation function. Let us briefly describe all these elements separately in the next sections.

\subsubsection{The influence matrix}

The influence matrix describes the geometric distribution of the thrusters. The physical distribution of the thrusters is presented in Figure 2. The influence matrices are then described as follows: 


$$
M_{1}=\left[\begin{array}{llll}
1 & -1 & -1 & 1
\end{array}\right] ; \quad M_{2}=\left[\begin{array}{llll}
1 & -1 & -1 & 1
\end{array}\right]
$$

Influence matrices $M_{1}$ and $M_{2}$ are associated to Satellite 1 and Satellite 2, respectively.

\subsubsection{Thruster saturation}

The saturation function is modeled by

$$
\operatorname{sat}_{(0, \bar{u})}\left(T_{(i)}\right)= \begin{cases}\bar{u}_{(i)} & \text { if } T_{(i)}>\bar{u}_{(i)} \\ T_{(i)} & \text { if } 0 \leq T_{(i)} \leq \bar{u}_{(i)}, i=1, \ldots, m . \\ 0 & \text { if } T_{(i)}<0\end{cases}
$$

The saturation bounds for the relative position control problem are $\underline{u}=0$ and $\bar{u}=$ $1 m N$, and $T=\left[T_{(1)}, \ldots, T_{(m)}\right]^{\prime}$ is the thrust applied on each satellite.

\subsubsection{Allocation function}

The allocation function (AF) used on real applications is a highly nonlinear optimized function. Indeed, finding an AF is a dynamic nonlinear optimization problem, which can be solved using non-linear optimization techniques [22], e.g., quadratic programming. Another option is to manipulate a simple nonlinear AF based on a switching structure. This nonlinear allocation function lies on the fact that the control output $y_{c}$ is treated component-wise, whereas the AF computes a set of thrust $T$ for each component of the control output. They are denoted by $y_{c(k)}$ and the set of thrusts associated is denoted by $T^{k}$. Finally, the thrust vector applied is the sum of all $T^{k}$ with $k=1, \ldots, m_{c}$. The switching structure can be described by the following expression:

$$
f\left(y_{c}\right)=\left\{\begin{array}{l}
T_{(i)}^{k}=\left\{\begin{array}{ll}
0 & \text { if } \operatorname{sign}\left(M_{(k, i)}\right) \neq \operatorname{sign}\left(y_{c(k)}\right) \\
\frac{y_{c(k)}}{\tau_{(k)} M_{(k, i)}} & \text { if } \operatorname{sign}\left(M_{(k, i)}\right)=\operatorname{sign}\left(y_{c(k)}\right)
\end{array}, k=1, \ldots, m_{c} .\right. \\
T_{(i)}=\sum_{k=1}^{m_{c}} T_{(i)}^{k}, i=1, \ldots, m . \\
T=\left[\begin{array}{lll}
T_{(1)} & \ldots & T_{(m)}
\end{array}\right]^{\prime}
\end{array}\right.
$$

where $\operatorname{sign}(\cdot)$ stands for the function sign and $\tau_{(k)}$ stands for the number of thrusters generating an effort of the same sign as $y_{c(k)} \cdot \tau_{(k)}$ is described as follows:

$$
\tau_{(k)}=\sum_{i=1}^{m}\left\{\operatorname{sign}\left(M_{(k, i)}\right)=\operatorname{sign}\left(y_{c(k)}\right)\right\}
$$

where $\left.\left\{\operatorname{sign}\left(M_{(k, i)}\right)=\operatorname{sign}\left(y_{c(k)}\right)\right)\right\}$ is a boolean function that returns 1 if both elements are equal or 0 if they are not. 
The relative position control problem presents $m=4$ thrusters and $k=1$ control output. Then the switching AF (7) has the following form for both satellites:

$$
f\left(y_{c}\right)=\left\{\begin{array}{c}
T_{(1)}=T_{(4)}=\left\{\begin{array}{l}
0 \text { if } y_{c}<0 \\
\frac{y_{c}}{2} \text { if } y_{c} \geq 0 \\
T_{(2)}=T_{(3)}=\left\{\begin{array}{l}
0 \\
0
\end{array} \text { if } y_{c} \geq 0\right. \\
\frac{y_{c}}{2} \text { if } y_{c}<0
\end{array}\right.
\end{array}\right.
$$

In that case the actuator is modeled as follows:

$$
u_{p}=\operatorname{Msat}_{(0, \bar{u})}\left(f\left(y_{c}\right)\right)=\left[\begin{array}{cc}
M_{1} & 0 \\
0 & M_{2}
\end{array}\right] \operatorname{sat}_{(0, \bar{u})}\left(\left[\begin{array}{l}
f\left(y_{c 1}\right) \\
f\left(y_{c 2}\right)
\end{array}\right]\right)
$$

Another AF is based on the pseudo-inverse matrix $M^{*}$ of the influence matrix $M$ and is given by

$$
T=f\left(y_{c}\right)=M^{*} y_{c}
$$

In this case the control input $u_{p}$ reads:

$$
u_{p}=\left[\begin{array}{cc}
M_{1} & 0 \\
0 & M_{2}
\end{array}\right] \operatorname{sat}_{(0, \bar{u})}\left(\left[\begin{array}{l}
M_{1}^{*} y_{c 1} \\
M_{2}^{*} y_{c 2}
\end{array}\right]\right)
$$

See also [7] where a multi-saturation based allocation function is suggested (together with an anti-windup approach that used [28]).

\subsection{Relative position closed-loop model}

With the previously presented plant (2), controller (3) and actuator (10), the closedloop system describing the relative position control reads:

$$
\left\{\begin{array}{l}
\dot{x}_{p}=A_{p} x_{p}+B_{p} u_{p}=\left[\begin{array}{ll}
0 & 1 \\
0 & 0
\end{array}\right]\left[\begin{array}{l}
\Delta z \\
\Delta \dot{z}
\end{array}\right]+\left[\begin{array}{cc}
0 & 0 \\
m_{1}^{-1} & -m_{2}^{-1}
\end{array}\right] u_{p} \\
\dot{x}_{c}=A_{c} x_{c}+B_{c} C_{p} x_{p} \\
y_{p}=C_{p} x_{p}=\left[\begin{array}{ll}
1 & 0
\end{array}\right] x_{p}=\Delta z \\
z_{p}=C_{z} x_{p}=\left[\begin{array}{ll}
1 & 0
\end{array}\right] x_{p}=\Delta z \\
u_{p}=M_{s a t}(0, \bar{u})\left(f\left(y_{c}\right)\right)=\left[\begin{array}{l}
M_{1} \operatorname{sat}_{(0, \bar{u})}\left(f\left(y_{c 1}\right)\right) \\
M_{2} \operatorname{sat}_{(0, \bar{u})}\left(f\left(y_{c 2}\right)\right)
\end{array}\right] \\
y_{c}=C_{c} x_{c}+D_{c} C_{p} x_{p}
\end{array}\right.
$$

with $f\left(y_{c}\right)$ defined by (9).

System (13) provides a benchmark for further simulations. However the nonlinearity introduced by the AF (9) makes complex the characterization of constructive conditions ${ }^{1}$ to compute the anti-windup compensator for this system. For this rea-

${ }^{1}$ in the sense that tractable numerical procedures can be associated to exhibit the matrices of the anti-windup compensator. 
son an alternative formulation can be used based on (12) leading to the closed-loop system:

$$
\left\{\begin{array}{l}
\dot{x}_{p}=A_{p} x_{p}+B_{p} u_{p}=\left[\begin{array}{ll}
0 & 1 \\
0 & 0
\end{array}\right]\left[\begin{array}{l}
\Delta z \\
\Delta \dot{z}
\end{array}\right]+\left[\begin{array}{cc}
0 & 0 \\
m_{1}^{-1} & -m_{2}^{-1}
\end{array}\right] u_{p} \\
\dot{x}_{c}=A_{c} x_{c}+B_{c} C_{p} x_{p} \\
y_{p}=C_{p} x_{p}=\left[\begin{array}{ll}
1 & 0
\end{array}\right] x_{p}=\Delta z \\
z_{p}=C_{z} x_{p}=\left[\begin{array}{ll}
1 & 0
\end{array}\right] x_{p}=\Delta z \\
u_{p}=M s a t_{(0, \bar{u})}\left(M^{*} y_{c}\right)=\left[\begin{array}{l}
M_{1} \operatorname{sat}_{(0, \bar{u})}\left(M_{1}^{*} y_{c 1}\right) \\
M_{2} \operatorname{sat}_{(0, \bar{u})}\left(M_{2}^{*} y_{c 2}\right)
\end{array}\right] \\
y_{c}=C_{c} x_{c}+D_{c} C_{p} x_{p}
\end{array}\right.
$$

\section{Anti-windup on the relative position control}

The relative position control problem has been modeled. Before dealing with the anti-windup computation purposes, let us consider the saturation function in (14). It is asymmetric. Since most of the results on anti-windup synthesis consider symmetric saturations, the saturation has to be symmetrized in order to adapt some of these results. To do that the symmetrizing technique of [8] is applied. More precisely, introducing the so-called symmetrizing vector $N \zeta_{\text {sym }}=\bar{u} / 2$, consider the scheme depicted in Figure 3.

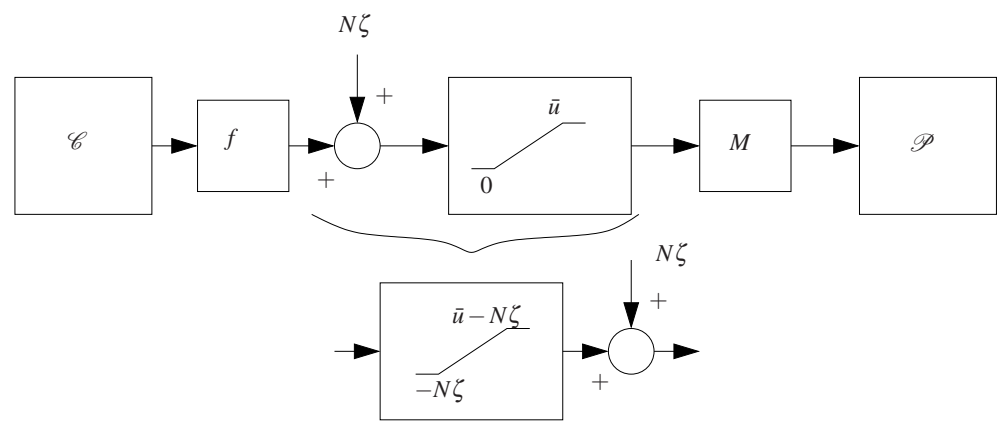

Fig. 3: Saturation bounds modification

Hence, we define

$$
N \zeta_{\text {var }}=\min \left(\max _{(i)}\left(\left|f\left(y_{c}\right)\right|\right), N \zeta_{\text {sym }(i)}\right)\left[\begin{array}{c}
1 \\
\vdots \\
1
\end{array}\right], i=1, \ldots, m
$$

which verifies that $M N \zeta_{v a r}=0$. That corresponds to a Variable Kernel Function (VKF) and solves the problems of extra consumption as exposed in [8]. Assuming 
the consumption to be proportional to the integral of all the thrust responses one can check that the actual consumption can be reduced by adequately defining the VKF. For more detail, the reader can consult [6]. With the saturation symmetrized, the closed-loop system (14) we want to study in order to design anti-windup loops can be written as:

$$
\left\{\begin{array}{l}
\dot{x}_{p}=A_{p} x_{p}+B_{p} u_{p}=\left[\begin{array}{ll}
0 & 1 \\
0 & 0
\end{array}\right]\left[\begin{array}{l}
\Delta z \\
\Delta \dot{z}
\end{array}\right]+\left[\begin{array}{cc}
0 & 0 \\
m_{1}^{-1} & -m_{2}^{-1}
\end{array}\right] u_{p} \\
\dot{x}_{c}=A_{c} x_{c}+B_{c} y_{p}+v_{x} \\
y_{p}=C_{p} x_{p}=\left[\begin{array}{ll}
1 & 0
\end{array}\right] x_{p}=\Delta z \\
z_{p}=C_{z} x_{p}=\left[\begin{array}{ll}
1 & 0
\end{array}\right] x_{p}=\Delta z \\
u_{p}=M s a t_{\left(u_{0}\right)}\left(M^{*} y_{c}\right)=\left[\begin{array}{l}
M_{1} \operatorname{sat}_{\left(u_{0}\right)}\left(M_{1}^{*} y_{c 1}\right) \\
M_{2} \operatorname{sat}_{\left(u_{0}\right)}\left(M_{1}^{*} y_{c 2}\right)
\end{array}\right] \\
y_{c}=C_{c} x_{c}+D_{c} C_{p} x_{p}+v_{y}
\end{array}\right.
$$

with symmetric bounds $u_{0}=\frac{1}{2} m N$ and where $v_{x}$ and $v_{y}$ are the output signals of the anti-windup compensator to be designed.

\subsection{Anti-windup compensator synthesis}

Three types of anti-windup compensators are investigated: the Direct Linear AntiWindup (DLAW), the Model Recovery Anti-windup (MRAW) and the Extended Model Recovery Anti-windup (EMRAW).

All the results developed in the Direct Linear Anti-Windup (DLAW) context are based upon the use of the dead-zone nonlinearities and associated modified sector conditions. Indeed, any system with saturation may be rewritten with dead-zone nonlinearity. Let us recall that the dead-zone function is defined by $\phi_{\left(u_{0}\right)}\left(y_{c}\right)=y_{c}-$ $\operatorname{sat}_{\left(u_{0}\right)}\left(y_{c}\right)$. In this context, the anti-windup compensator is defined as follows:

$$
\mathscr{A} \mathscr{W}\left\{\begin{array}{l}
\dot{x}_{a w}=A_{a w} x_{a w}+B_{a w} \phi_{\left(u_{0}\right)}\left(y_{c}\right) \\
v_{x}=\left[\begin{array}{ll}
I_{n_{c}} & 0
\end{array}\right]\left(C_{a w} x_{a w}+D_{a w} \phi_{\left(u_{0}\right)}\left(y_{c}\right)\right) \\
v_{y}=\left[\begin{array}{ll}
0 & I_{m}
\end{array}\right]\left(C_{a w} x_{a w}+D_{a w} \phi_{\left(u_{0}\right)}\left(y_{c}\right)\right)
\end{array}\right.
$$

where $x_{a w} \in \mathfrak{R}^{n_{a w}}$ is the anti-windup state, $n_{a w} \geq 0, \phi_{\left(u_{0}\right)}\left(y_{c}\right)$, the dead-zone output is the anti-windup input, $v=\left[\begin{array}{ll}v_{x}^{\prime} & v_{y}^{\prime}\end{array}\right]^{\prime} \in \mathfrak{R}^{n_{c}+m}$ is the anti-windup output and $A_{a w}$, $B_{a w}, C_{a w}, D_{a w}$ are matrices of appropriate dimensions. Figure 4 presents the block diagram describing the DLAW structure [14], [27].

In the sequel, we consider by simplicity that $v_{y}=0$ to avoid implicit loop and therefore we consider a dynamic DLAW into the form:

$$
\mathscr{A} \mathscr{W}\left\{\begin{array}{l}
\dot{x}_{a w}=A_{a w} x_{a w}+B_{a w} \phi_{\left(u_{0}\right)}\left(y_{c}\right) \\
v_{x}=C_{a w} x_{a w}+D_{a w} \phi_{\left(u_{0}\right)}\left(y_{c}\right)
\end{array}\right.
$$




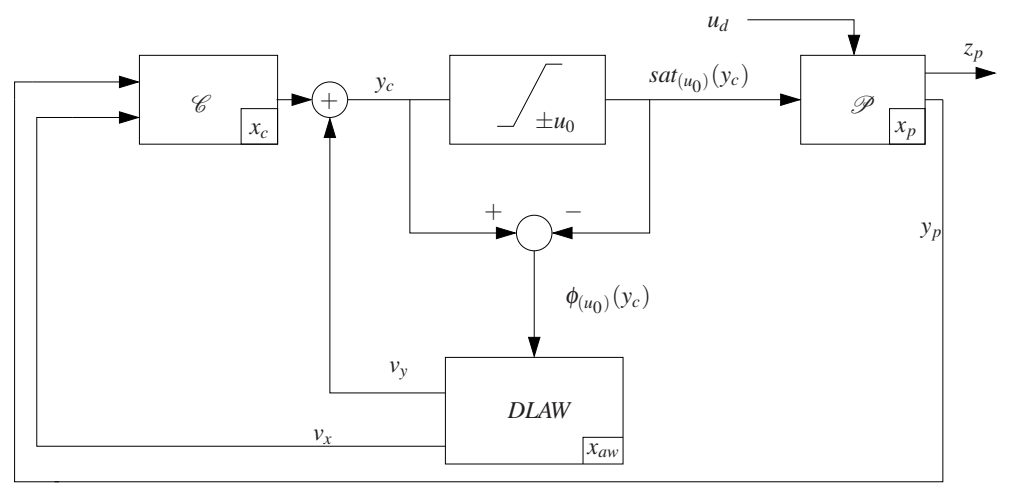

Fig. 4: Direct Linear Anti-Windup Structure.

where $x_{a w} \in \Re^{n_{a w}}$ is the anti-windup state, $n_{a w} \geq 0, v_{x} \in \mathfrak{R}^{n_{c}}$ is the anti-windup output and $A_{a w}, B_{a w}, C_{a w}, D_{a w}$ are matrices of appropriate dimensions.

\subsubsection{Static DLAW synthesis}

In the static case one chooses $n_{a w}=0$ and anti-windup matrices are null except $D_{a w}\left(A_{a w}=0, B_{a w}=0, C_{a w}=0\right)$. Thus, the anti-windup output is given by $v_{x}=$ $D_{a w} \phi_{\left(u_{0}\right)}\left(y_{c}\right)$ and $v_{y}=0$ [16].

The DLAW static gain $D_{a w}$ can be computed by using Lyapunov theory. In particular, in order to characterize a domain of asymptotic stability for the closed-loop system (including the plant, the controller with the static anti-windup loop and the linear closed-loop system without saturation) a quadratic Lyapunov function can be used to express some conditions in terms of Linear Matrix Inequalities (LMIs). The main advantages of using quadratic Lyapunov function resulting into LMIs reside in the fact that the synthesis of the anti-windup loop can then be carried out through the solution of convex optimization problems, which can be solved by efficient software packages [3], [21]. Hence, the stability domain can be optimized in some direction of interest, namely in the $\Delta z$ direction. In this case, by using the conditions developed in [6] (see Theorem 3.1 in Chapter 3 of [6]), the static anti-windup gain $D_{a w}$ obtained is:

$$
D_{a w}=\left[\begin{array}{cccccccc}
-0.32 & -0.32 & 0.32 & 0.32 & -1.65 & -1.65 & 1.65 & 1.65 \\
0.28 & 0.28 & -0.28 & -0.28 & 3.5 & 3.5 & -3.5 & -3.5 \\
2.95 & 2.95 & -2.95 & -2.95 & -6.39 & -6.39 & 6.39 & 6.39 \\
0.88 & 0.88 & -0.88 & -0.88 & -1.81 & -1.81 & 1.81 & 1.81 \\
376.78 & 376.78 & -376.78 & -376.78 & -665.5 & -665.5 & 665.5 & 665.5
\end{array}\right]
$$

Regarding the structure of the static DLAW (19), we can observe the difference between the first four columns and the last four ones. That is due to the relation of the 
first four columns with the first satellite thrusters, and the relation of the last four ones with the second satellite thrusters. In addition, it is important to remark that there is a row (the fifth one) whose values outstand in comparison with the others. Actually this line affects the state of the controller related to the integration. The anti-windup mission is to attenuate the integral state of the controller which is the more sensible state to the saturation effects. Therefore it is normal to find a more important effect on this controller state than on the others. With this anti-windup technique, we thus recover the usual method of de-charging the integral state, when a saturation occurs.

\subsubsection{Dynamic DLAW synthesis}

Using the results in [25], the matrices $A_{a w}, B_{a w}, C_{a w}, D_{a w}$ of the dynamic antiwindup compensator (18) can be computed by solving some LMIs when we choose the order of the anti-windup compensator $n_{a w}=n_{p}+n_{c}+n_{l}$. For more details see Propositions 3.1 and 3.2 in Chapter 3 of [6]. Nevertheless, in some cases the order of this anti-windup compensator can be too large to be implemented in a real onboard calculator. Thus, the goal now is to find a low order anti-windup compensator. In this case, the main difficulty resides in the choice of matrices $A_{a w}$ and $C_{a w}$, the matrices $B_{a w}$ and $D_{a w}$ remaining linearly computationable in the conditions. A way to do this is to use the full order anti-windup compensator computation as a guide on the choice of the poles for the fixed order anti-windup synthesis. The computation is decomposed in two steps. The first one consists in computing the full order DLAW. From all the poles of the computed full order compensator, only the poles of $A_{a w}$ sharing the same magnitude order as those of the linear closed-loop system without saturation (named $A_{l}$ ) are retained (see also $[5,9]$ ). The second step consists in testing the conditions to obtain $B_{a w}$ and $D_{a w}, A_{a w}$ and $C_{a w}$ being fixed from the first step (see, for example, [20, 27]).

As in the static anti-windup case, the stability domain is maximized in the relative position direction $\Delta z$. Table 1 shows the poles of the full order dynamic DLAW and the poles of the linear closed-loop system $A_{l}$. The selected poles for the fixed order DLAW synthesis are marked with $*$.

\subsubsection{MRAW and EMRAW synthesis}

The Model Recovery Anti-Windup (MRAW) consists in selecting the anti-windup compensator as a dynamical filter, incorporating a model of the plant [14]. The aim of this filter is to recover the unconstrained closed-loop dynamics. The plant control is limited by the saturation nonlinearity, thus, recovering the missing control and filtering it throughout the anti-windup compensator we can recover the missing dynamics of the plant. This recovered dynamics allows the system to keep tracking what the closed-loop response would be in the absence of saturation. The equations describing filter dynamics are stated by: 
Table 1: Full order DLAW eigenvalues in relative position control

\begin{tabular}{cc}
\hline $\operatorname{eig}\left(A_{a w}\right)(* \equiv$ selected $)$ & $\operatorname{eig}\left(A_{l}\right)$ \\
\hline$-8.28 \cdot 10^{6}$ & $(-2.61 \pm j 2.88) \cdot 10^{-1}$ \\
$-6.21 \cdot 10^{6}$ & $(-1.62 \pm j 2.02) \cdot 10^{-1}$ \\
$-4.74 \cdot 10^{4}$ & $(-8.23 \pm j 8.23) \cdot 10^{-3}$ \\
$-1.93 \cdot 10^{2}$ & $-2.73 \cdot 10^{-3}$ \\
-1.61 & \\
$(-9.11 \pm j 27.5) \cdot 10^{-2}$ & \\
-0.13 & \\
$-4.38 \cdot 10^{-2}(*)$ & \\
$(-8.97 \pm j 5.17) \cdot 10^{-3}(*)$ & \\
$-8.61 \cdot 10^{-3}(*)$ & \\
$-7.46 \cdot 10^{-3}(*)$ & \\
$-4.11 \cdot 10^{-3}$ & \\
\hline
\end{tabular}

$$
\left\{\begin{aligned}
\dot{x}_{a w} & =A_{p} x_{a w}+B_{p}\left(s a t_{u_{0}}\left(y_{c}+v_{1}\right)-y_{c}\right) \\
y_{a w} & =C_{p} x_{a w} \\
v_{1} & =g\left(x_{a w}\right)
\end{aligned}\right.
$$

where $x_{a w} \in \mathfrak{R}^{n_{p}}$ is the anti-windup compensator state, $y_{a w} \in \mathfrak{R}^{q}$ and $v_{1} \in \mathfrak{R}^{m}$ its outputs, and $g$ is nonlinear function suitably designed ([30, 32]). The anti-windup compensator structure is represented in Figure 5. Notice that, by using the DLAW

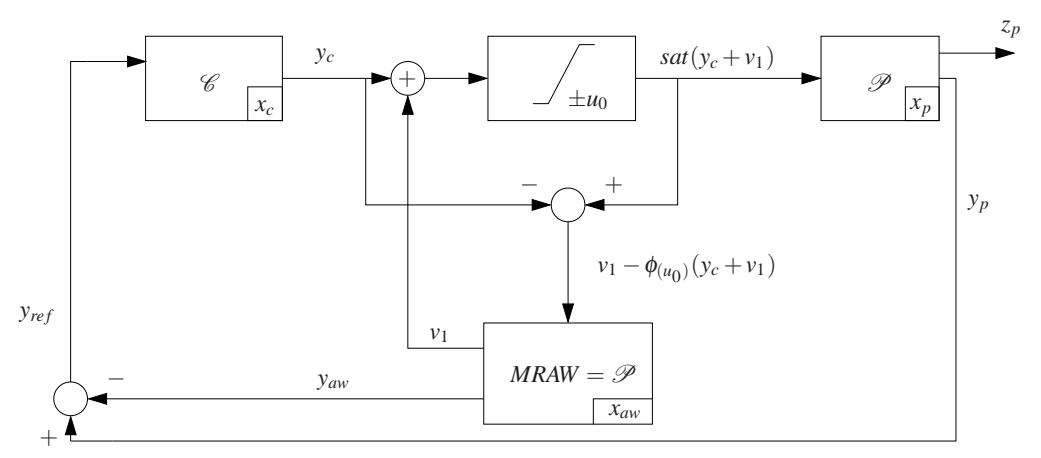

Fig. 5: MRAW control block diagram

framework, the anti-windup loop is injected to the controller by considering $v_{x}$ and $v_{y}$ as follows:

$$
\begin{aligned}
& v_{x}=-B_{c} y_{a w} \\
& v_{y}=-D_{c} y_{a w}+v_{1}
\end{aligned}
$$

where $v_{x}$ and $v_{y}$ are described by Figure 4 . 
A first possible solution to the MRAW problem is to select $v_{1}$ as a linear state feedback from $x_{a w}$ designed completely disregarding the saturation effects. These solutions are associated to local stability properties but, for exponentially stable plants, the global stability is possible [13]. Another type of solutions that one can propose within the MRAW compensation is to select $v_{1}$ as a nonlinear function of the anti-windup compensator state $x_{a w}$. This type of solution is certainly the most difficult to design and to implement but it is the most advanced scheme within this framework. Reference [12] gives constructive conditions to find such a stabilizing law $v_{1}$. See also [14], [27], [33] and references therein.

The Extended Model Recovery Anti-Windup (EMRAW) techniques is a combination of both the DLAW and MRAW structures, as depicted in Figure 6.

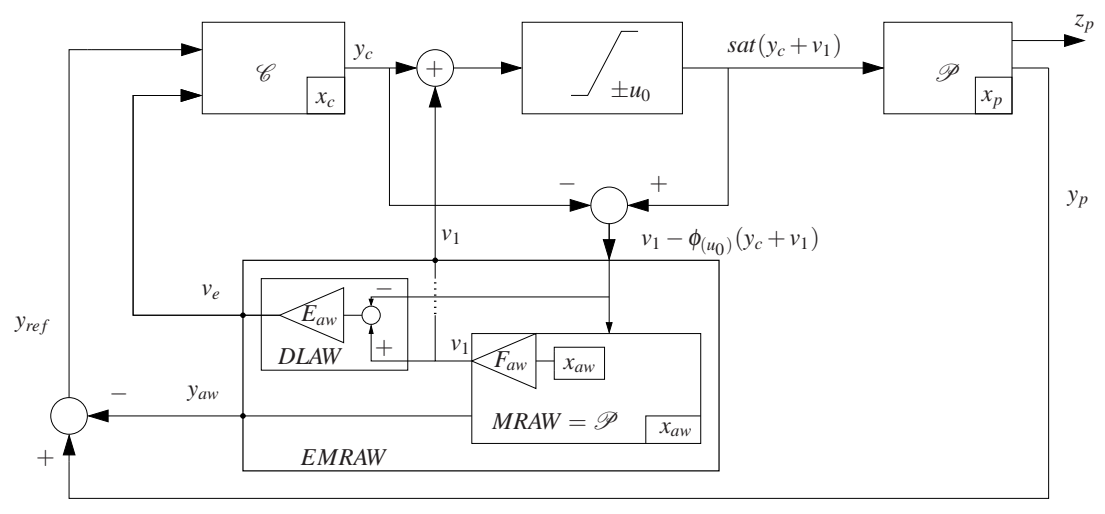

Fig. 6: Extended MRAW block diagram.

The EMRAW strategy is then described by the following equations:

$$
\mathscr{A} \mathscr{W}:\left\{\begin{array}{l}
\dot{x}_{a w}=\left(A_{p} x_{a w}+B_{p} v_{1}\right)+B_{p} \phi_{\left(u_{0}\right)}\left(y_{c}+v_{1}\right) \\
y_{a w}=C_{p} x_{a w} \\
v_{1}=F_{a w} x_{a w} \\
v_{e}=E_{a w} \phi_{\left(u_{0}\right)}\left(y_{c}+v_{1}\right)
\end{array}\right.
$$

where $x_{a w} \in \Re^{n_{a w}}$ is the anti-windup compensator state with $n_{a w}=n_{p}, y_{a w} \in \Re^{q}$ and $v_{1} \in \mathfrak{R}^{m}$ are the outputs generated by MRAW stage and $v_{e} \in \mathfrak{R}^{n_{c}}$ is the output issued by the static DLAW stage (gain $E_{a w}$ ). Notice $E_{a w}$ is an static DLAW as it feedbacks the dead-zone function $\phi_{\left(u_{0}\right)}$ directly into the controller dynamics $x_{c}$ through the signal $v_{e}$. First an objective-based algorithm, as in [5], (or a coordinate-descending algorithm as in [23]) is applied to compute some gains, and then some stability conditions are solved to compute the anti-windup loop. See [6, 9] for more details, and for the numerical data of this design. 


\subsection{Simulations on relative position control}

First, the closed-loop system (13) is simulated without anti-windup. System (13) is simulated from an initial condition of $x_{p}(0)=\left[\begin{array}{ll}\Delta z & \Delta \dot{z}\end{array}\right]^{\prime}=\left[\begin{array}{ll}-1 & 0\end{array}\right]^{\prime}$ and $x_{c}=0_{1 \times 5}$. Let us remind that (13) describes the relative position closed-loop system with the switching AF (9).
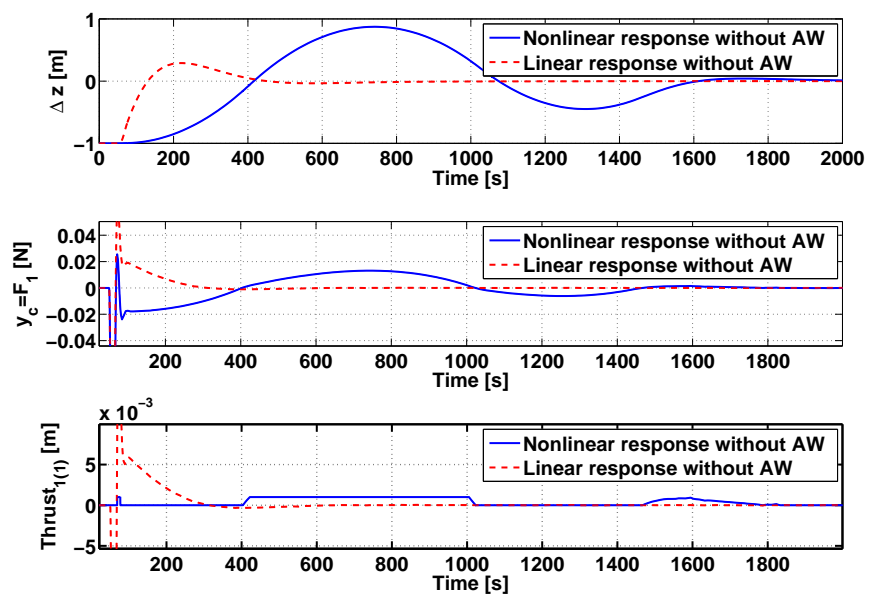

Fig. 7: Responses of the relative position without anti-windup compensator.

In Figure 7, the solid line presents the $\Delta z$ response of the system (13) (i.e. nonlinear response). The dot-dashed line shows the response of the system without saturation (i.e. linear response). One can realize the effects of the saturation as oscillations are induced in the nonlinear response of $\Delta z$. The control output and the performed thrust are also shown in Figure 7. The thrust response is saturated and the control output oscillates. The effects of the saturation on the system (13) fully justify the introduction of the anti-windup compensator.

In Figure 8, the time evolution of the relative position of both satellites using a compensator developed by Thales Alenia Space (TAS), Cannes, France is shown. It can be observed that this ad-hoc compensator succeeds in avoiding the oscillations, even in presence of the nonlinearities in the closed loop. However, whereas this control law performs very well in single-input single-output control problems, it cannot be easily generalized to multivariable systems, and thus to the non simplified formation flying control problem for which relative position and attitude are coupled. This is an additional motivation to develop the anti-windup techniques presented in this chapter.

\footnotetext{
${ }^{2}$ Throughout the simulations, the controller state is considered to be always initialized at the origin.
} 

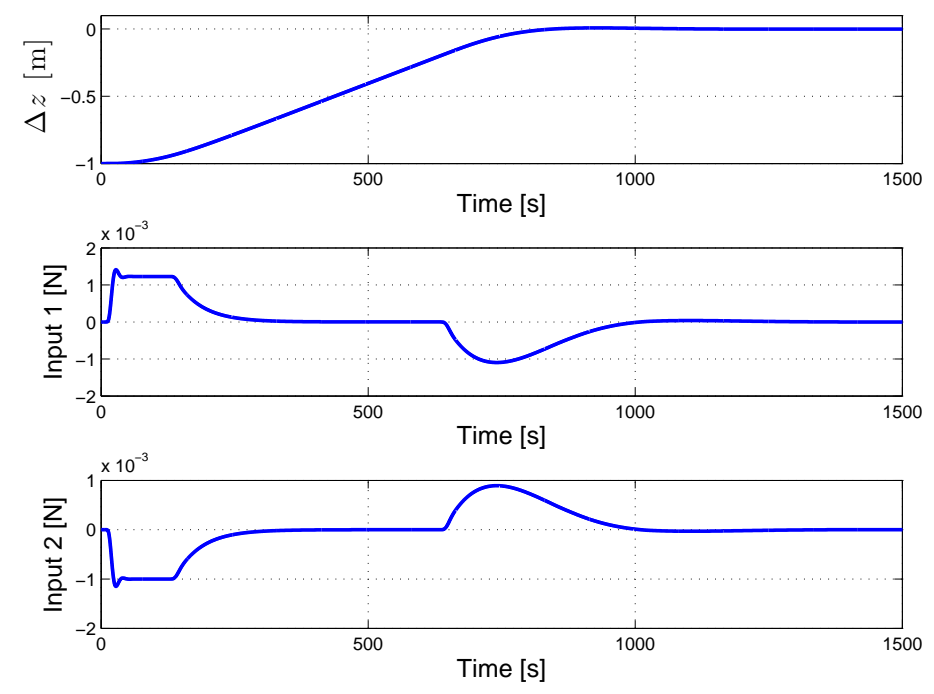

Fig. 8: Responses of the relative position with compensator developed by TAS. Top: relative position of both satellites. Middle: input of the first satellite. Down: input of the second satellite.

The closed-loop system (16) is then simulated with the following anti-windup compensators:

- static DLAW compensator (solid line);

- full order dynamic DLAW compensator (dashed line);

- fixed order dynamic DLAW compensator (dot-dashed line);

- MRAW compensator (line with dots);

- EMRAW compensator computed with a coordinate-descending algorithm that is denoted Alg. 3.2 (line with circles);

- EMRAW compensator computed with an objective-based algorithm, that is denoted Alg. 3.3 (line with stars).

Figure 9 shows the response of the relative position with the different anti-windup compensators in the control loop. The $\Delta z$ responses have been split in two small figures for clarity purposes. From a general overview of the Figure 9 one can conclude that the oscillation observed in Figure 7 has disappeared with the introduction of the anti-windup compensator.

Let us start analyzing the response with DLAW. In Figure 9 the response with a dynamic DLAW is smoother than in the static DLAW case which presents a drop on its slope. The anti-windup action, in the static case, ends as soon as there is no saturation. Therefore the drop appears. On the contrary, the dynamic DLAW keeps modifying the controller action even without actuator saturation. In Figure 10 the thrust response suddenly falls with the static DLAW case while it decreases 


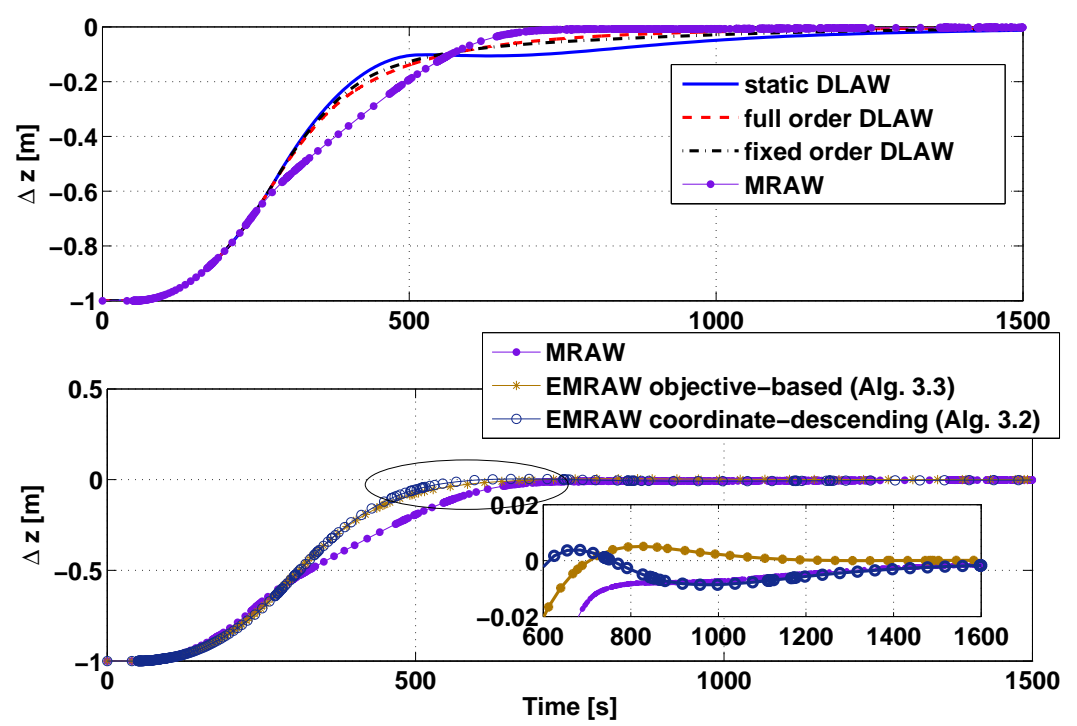

Fig. 9: Response of the relative position with several anti-windup compensators.

progressively with the dynamic one. The advantage of a dynamic DLAW is then proven.

Also in Figure 10, the thrust response with a full order DLAW is noisy. The fast dynamics in the full order case induces the high frequency oscillation. The presence of fast and slow modes in the full order DLAW generates numerical problems for both LMI computation step (bad conditioning effects) and simulation step (numerical precision effects). On the contrary, the fixed order DLAW does not present this oscillation because the fast dynamics were not chosen in the synthesis process. Therefore, the fixed order provides a smooth response without high frequency oscillations.

Let us analyze in Figure 9 the $\Delta z$ response with a MRAW/EMRAW: actually, fast responses are attained. Moreover the response $\Delta z$ of the system (16) is faster with the EMRAW. An explanation to this behavior is find in Figures 11 and 12 where the anti-windup output $y_{a w}$ and the reference signal $y_{\text {ref }}$ are compared. The system response with the MRAW attains the reference, that is, $y_{p}=y_{\text {ref }}$ and $y_{a w}=0$, before the response with the EMRAW. However the reference $y_{r e f}$ for the MRAW is slower than the one for the EMRAW. Therefore, the actual response $y_{p}=\Delta z$ of the system (16) is faster with the EMRAW.

Another comment on the MRAW approach can be done by the analysis of the thrust response. In Figure 10 large oscillations on the MRAW approach can be noticed. However the closer it gets to the origin the higher the bang-bang frequency and hence the stronger the noise induced into the thrust action. 


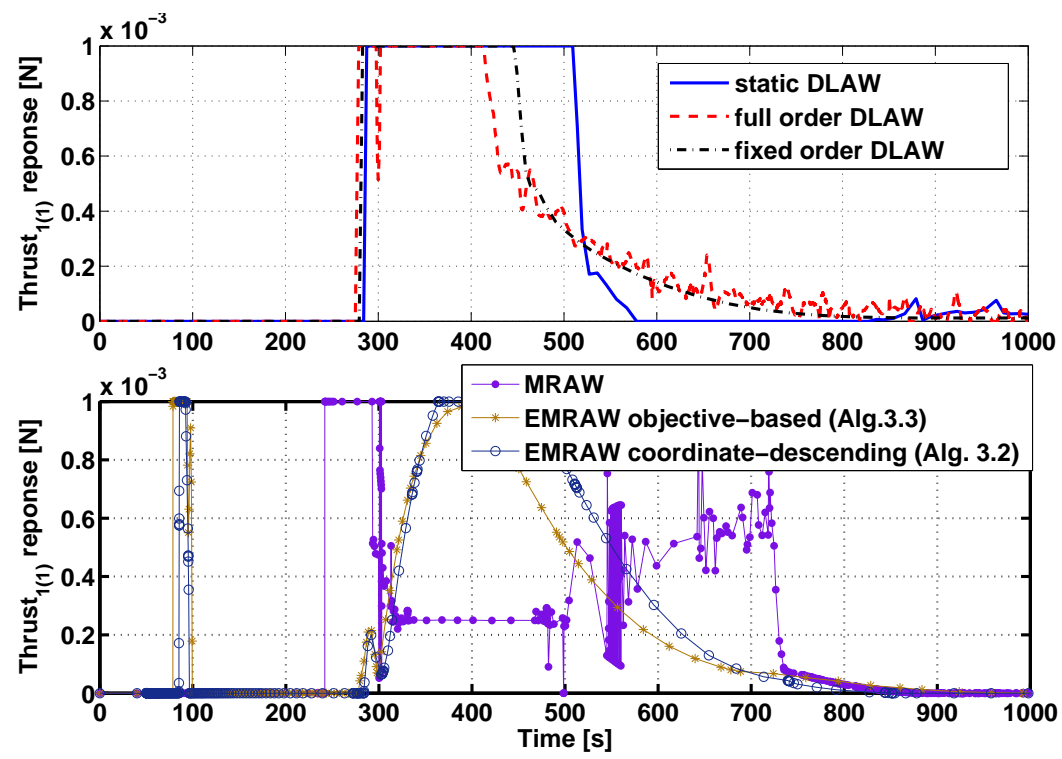

Fig. 10: Response of the first thruster with several Anti-windup compensators.

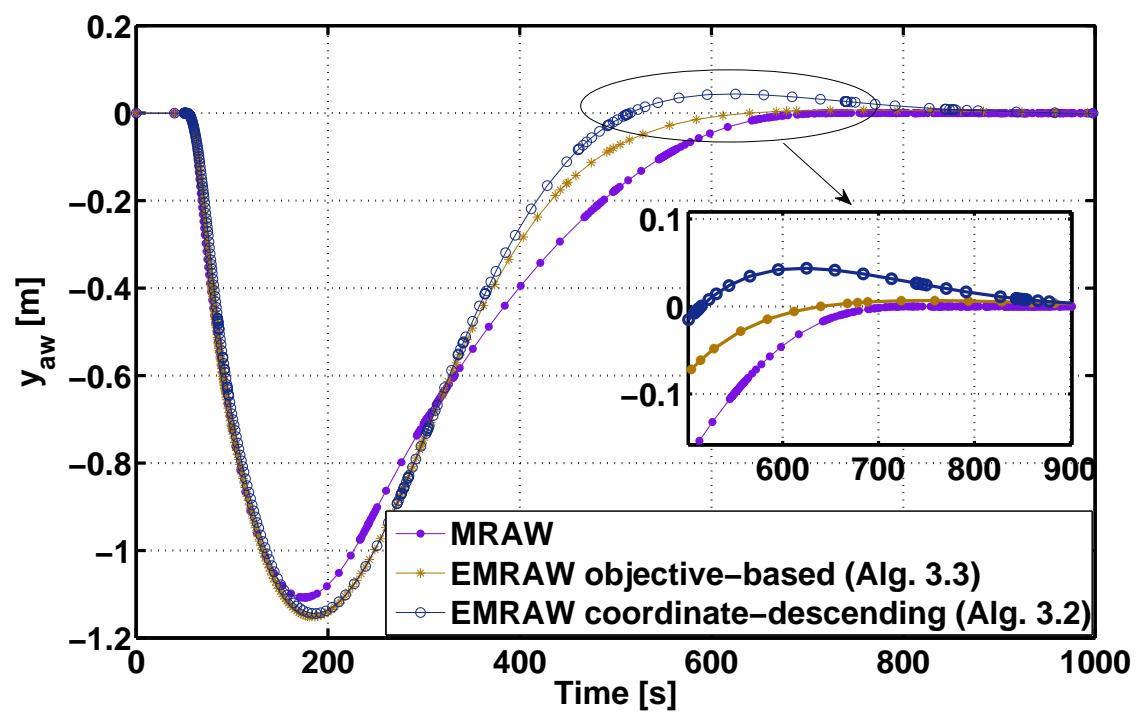

Fig. 11: Anti-windup output response for MRAW/EMRAW approaches.

Finally, Figure 13 presents the stability domain estimation for the different antiwindup compensators. The MRAW case has been omitted. 


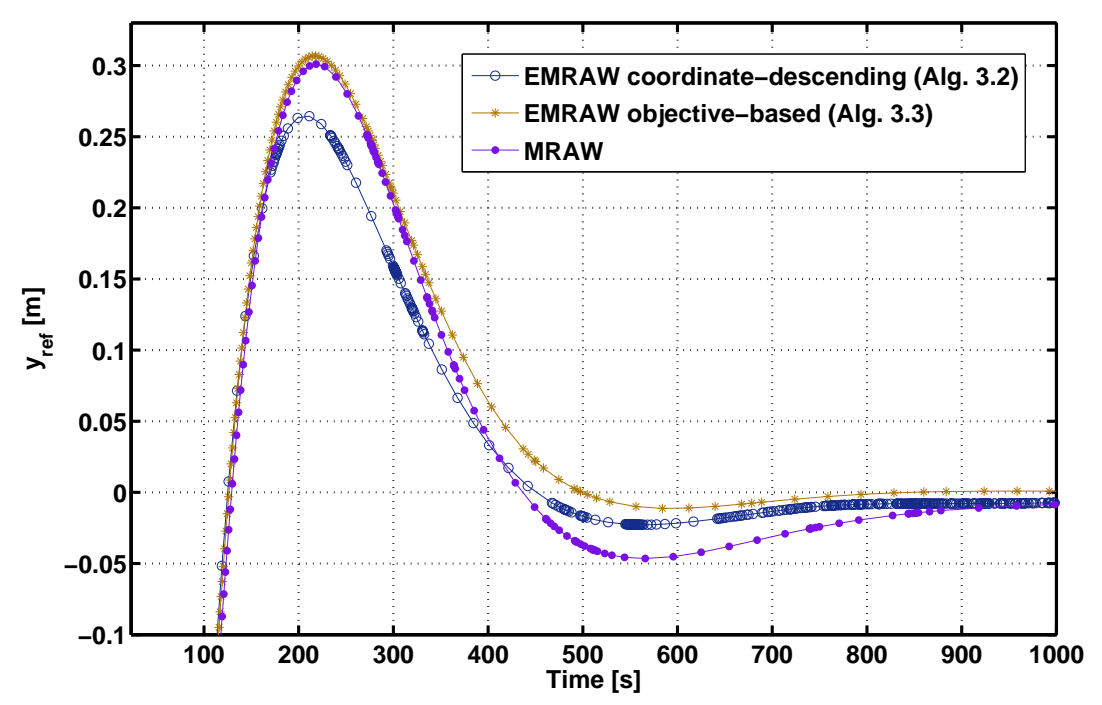

Fig. 12: Reference response for MRAW/EMRAW approaches.

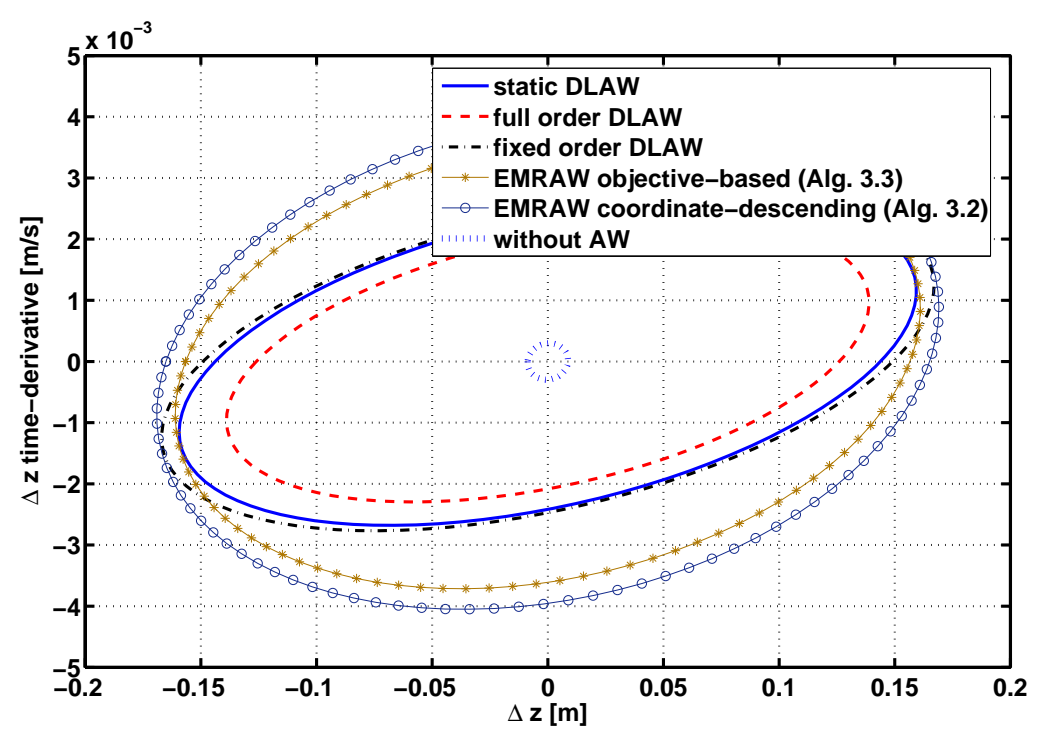

Fig. 13: Stability domain with several anti-windup compensators.

The EMRAW computed with the coordinate-descending algorithm (based on [23]) provides a larger estimation. Despite slight differences, all the compensators 
ensure (more or less) the same estimated maximal admissible $\Delta z_{\max }(0)$ (cutting point with $\Delta \dot{z}=0$ axis). However, the estimation obtained with the full order DLAW is smaller than the one obtained with the other compensators. This is due to some numerical problems. The difference between the maximum and the minimum eigenvalue of $A_{a w}$ results on a bad conditioning of the LMIs conditions. As a consequence, the stability domain analysis for the full order DLAW is unfeasible.

Let us make two final remarks on the stability domain estimation. First notice that the size of the stability domain without anti-windup (dots line) is clearly smaller. The second point is the conservatism of the estimation. Simulations show that the system is stable (even without anti-windup) for an initial condition $x_{p}(0)=\left[\begin{array}{ll}-1 & 0\end{array}\right]^{\prime}$. However, considering the estimated domain the maximal admissible initial condition is around $x_{p}(0)=\left[\begin{array}{ll}-0.15 & 0\end{array}\right]^{\prime}$.

Table 2: Summary values on the relative position control

\begin{tabular}{c|ccc}
\hline & Static DLAW & full order DLAW & fixed order DLAW \\
\hline$\Delta z_{\max }(0)=\frac{1}{\sqrt{\rho}}[\mathrm{m}]$ & 0.14 & 0.130 .15 & \\
Time of response $[s]$ & 1570 & 1160 & 1390 \\
Consumption $[N s]$ & 2.017 & 1.829 & 1.82 \\
AW Order & 0 & 14 & 5 \\
\hline$\Delta z_{\max }(0)=\frac{1}{\sqrt{\rho}}[\mathrm{m}]$ & MRAW & EMRAW Algo. 3.2 EMRAW Algo. 3.3 \\
Time of response $[s]$ & 750 & 0.17 & 0.16 \\
Consumption $[N s]$ & 1.679 & 580 & 630 \\
AW Order & 2 & 1.894 & 1.8192 \\
& & 2 & 2
\end{tabular}

Table 2 summarizes the main values characterizing the anti-windup compensators. These values are:

- the maximal admissible initial condition for the relative position $\Delta z_{\max }(0)$;

- the time evolution for $\Delta z$ in seconds;

- the integral of the thrust response (value related to the consumption);

- the order of the anti-windup (AW) compensator.

A certain trade-off has to be done in the optimized compensator design between performance (consumption, speed of convergence) and size of the stability domain $(\rho)$.

The time evolution appears as a remarkable value for the comparison. It has been defined as the time when the $\Delta z$ response has reached the $99 \%$ of the gap between its initial condition and the origin. In these simulations the initial relative position is $\Delta z=-1 \mathrm{~m}$. It has been understood as $\Delta z= \pm 0.01 \mathrm{~m}$.

Note the important gap between the time evolution of the system with a DLAW or with a MRAW/EMRAW. With these last approaches the responses are certainly faster. In addition, this improvement does not come with an undesirable increase of the consumption. 
Let us remind that the value representing the consumption is just the integral of the thrusters response with relation to the time, and not the actual consumption. These values are however proportional.

Remark 2. Because of its simplicity, its efficiency in terms of time evolution and its guaranteed stability domain, the EMRAW structure arises as an interesting architecture for the anti-windup computation. However the initialization process for the associated algorithms is not trivial. In addition, the anti-windup compensator order is restricted to the order of the plant.

Remark 3. The full order DLAW presents fast and slow modes. These modes generate numerical problems for both LMI computation step (bad conditioning effects) and simulation step (numerical precision effects). Therefore, the fixed order DLAW is an interesting alternative to the full order one. Although some strategies are proposed in the literature, the choice of the $A_{a w}$ poles is not strictly formalized.

Remark 4. Even if in Table 2 the static DLAW presents the worst values of all compensators, these values are not too much far from those obtained with other approaches. On the other hand, the static DLAW is easy to compute because of the associated LMIs simplicity. It is also easy to implement because it is simply a static gain. Therefore, a systematic procedure can be designed for the static DLAW computation.

\section{Conclusion}

Anti-windup compensator design represents the core of this chapter. For ease of understanding and methods comparisons, the relative position control system has been taken as a simplified study case. Different anti-windup compensators have been evaluated for this problem. The closed-loop system modeling this control problem has been initially provided. The simplicity of the system has allowed the characterization of the main features of the anti-windup compensators. The simulations have allowed to point out the interest of the EMRAW approach. Similarly to the study presented in this chapter, the attitude and relative position control system has also been studied. This application presents the particularity of being a multi-variable system with a coupling of the attitude over the relative position. For more details on this last case see [6].

Interesting point for further research, regarding the EMRAW, concerns on the anti-windup order reduction. Indeed, one main drawback of the EMRAW is that the plant and the anti-windup compensator are forced to have the same order. In large order models, this constraint is unacceptable for future physical implementations. Therefore, it would be interesting to develop constructive methods to reduce the anti-windup order, for example, eliminating secondary dynamics of the considered plant. In this scenario, the results could be related with the anti-windup synthesis on an uncertain system. 


\section{References}

1. O. Absil. Science with pegase. In Proceedings of $2^{\text {nd }} T P F /$ Darwin International Conference, San Diego, CA, USA, July, 2004.

2. K.J. Aström and L. Rundqwist. Integrator windup and how to avoid it. In Proceedings of the American Control Conference, Pittsburgh, PA, USA, 1989.

3. G. Balas, R. Chiang, A. Packard, and M. Safonov. Robust Control Toolbox user's guide. The MathWorks Inc., 2007.

4. J.M. Berg, K.D. Hammet, C.A. Schwartz, and S.S. Banda. Analysis of the destabilizing effect of daisy chained rate-limited actuators. IEEE Transactions on Control Systems Technology, 4:171-176, 1996.

5. J.M. Biannic, C. Roos, and S. Tarbouriech. A practical method for fixed-order anti-windup design. In Proceedings of $7^{\text {th }}$ IFAC Symposium on Nonlinear Control Systems (NOLCOS), Pretoria, South Africa, August, 2007.

6. J. Boada. Satellite control with saturating inputs. PhD thesis, ISAE, Toulouse, France, 2010.

7. J. Boada, C. Prieur, S. Tarbouriech, C. Pittet, and C. Charbonnel. Multi-saturation anti-windup structure for satellite control. In Proceedings of the American Control Conference, Baltimore, MD, USA, July, 2010.

8. J. Boada, C. Prieur, S. Tarbouriech, C. Pittet, and C. Charbonnel. Anti-windup design for satellite control with microthrusteurs. In Proceedings of AIAA GN\&C conference, Chicago, IL, USA, August, 2009.

9. J. Boada, C. Prieur, S. Tarbouriech, C. Pittet, and C. Charbonnel. Extended model recovery anti-windup for satellite control. In Proceedings of the $16^{\text {th }}$ IFAC Symposium on Automatic Control in AeroSpace, Nara, Japan, September, 2010.

10. S. Boyd and L. S.P.Vandenberghe. Convex optimization. Cambridge Univesity Press, 2004.

11. D.C. Durham. Constrained control allocation. AIAA Journal of Guidance, Control and Dynamics, 16:717-725, 1993.

12. A.R. Galeani, A.R. Teel, and L. Zaccarian. Constructive nonlinear anti-windup design for exponentially unstable linear plants. Systems and Control Letters, 56(5):357-365, 2007.

13. S. Galeani, A.R. Onori, A.R. Teel, and L. Zaccarian. Regional, semiglobal, global nonlinear anti-windup via switching design. In Proceedings European Control Conference, Kos, Greece, July, 2007.

14. S. Galeani, S. Tarbouriech, M.C. Turner, and L. Zaccarian. A tutorial on modern anti-windup design, volume 15(3-4):418-440. European Journal of Control, 2009.

15. S. Gaulocher. Commande boucle fermée multivariable pour le vol en formation de vaisseaux spatiaux. PhD thesis, Université de Toulouse, Toulouse, France, 2007.

16. J.M. Gomes da Silva Jr. and S. Tarbouriech. Anti-windup design with guaranteed regions of stability: An LMI-based approach. IEEE Transactions on Automatic Control, 50(1):106-111, 2005.

17. G. Grimm, J. Hatfield, I. Postlethwaite, A.R. Teel, M.C. Turner, and L. Zaccarian. Antiwindup for stable linear systems with input saturation: an LMI-based synthesis. IEEE Transactions on Automatic Control, 48(9):1509-1525, 2003.

18. T. Hu and Z. Lin. Control systems with actuator saturation. Analysis and design. Birkhauser, 2001.

19. V. Kapila and K. Grigoriadis (Eds.). Actuator saturation control. Marcel Dekker, Inc., 2002.

20. M.L. Kerr, M.C. Turner, and I. Postlethwaite. Practical approaches to low-order anti-windup compensator design: a flight control comparison. In IFAC World Congress, Seoul, Korea, 2008.

21. J. Löfberg. Yalmip : A toolbox for modeling and optimization in matlab. In CACSD Conference, Taipei, Taiwan, 2004.

22. J. Nocedal and S.J. Wright. Numerical optimization. New York: Springer-Verlag, 1999.

23. D. Peaucelle and D. Arzelier. An efficient numerical solution for $\mathrm{H}_{2}$ static output feedback synthesis. In Proceedings of European Control Conference, Porto, Portugal, 2001. 
24. L. Pirson, C. Charbonnel, B. Udrea, M. Rennie, P. McGuinness, and P. Palomo. Darwin precursor demonstration mission: The ICC2 study, from GNC design to real-time test bench validation. In Proceedings of $6^{\text {th }}$ GNC ESA, Loutraki, Greece, 2005.

25. C. Roos and J.M. Biannic. A convex characterization of dynamically-constrained anti-windup controllers. Automatica, 44(8):2449-2452, 2008.

26. G. Stein. Bode lecture: respect the unstable. In Proceedings of Conference on Decision and Control, Tampa, USA, December, 1989.

27. S. Tarbouriech, G. Garcia, J.M. Gomes da Silva Jr., and I. Queinnec. Stability and stabilization of linear systems with saturating actuators. Springer, London, 2011.

28. S. Tarbouriech, C. Prieur, and J.M. Gomes da Silva Jr. Stability analysis and stabilization of systems presenting nested saturations. IEEE Transactions on Automatic Control, 51(8):13641371, 2006.

29. S. Tarbouriech and M.C. Turner. Anti-windup synthesis: an overview of some recent advances and open problems. IET Control Theory and Application, 3(1):1-19, 2009.

30. A.R. Teel and N. Kapoor. The $\mathscr{L}_{2}$ antiwindup problem: its definition and solution. In Proceedings of the $4^{\text {th }}$ European Control Conference, Brussels, Belgium, July, 1997.

31. M.C. Turner and I. Postlethwaite. A new perspective on static and low order anti-windup synthesis. International Journal of Control, 77(1):27-44, 2004.

32. L. Zaccarian and A.R. Teel. A common framework for anti-windup, bumpless transfer and reliable designs. Automatica, 38(10):1735-1744, 2002.

33. L. Zaccarian and A.R. Teel. Modern anti-windup synthesis. Princeton University Press, Princeton, 2011. 